\begin{tabular}{|c|c|c|}
\hline Revista do Departamento de Geografia USP & $\begin{array}{l}\text { Revista do Departamento de Geografia } \\
\text { Universidade de São Paulo } \\
\text { www.revistas.usp.br/rdg } \\
\text { ISSN 2236-2878 } \\
\text { V.34 (2017) }\end{array}$ & 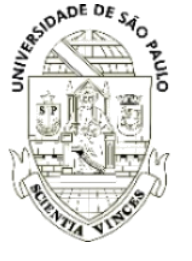 \\
\hline
\end{tabular}

\title{
Reflexões Acerca do Comércio e Consumo na Perspectiva da Geografia Humana
}

\author{
Reflections About the Commerce and Consumption In \\ Perspective of The Human Geography
}

\author{
Tiago Estevam Gonçalves \\ Instituto Federal de Educação Ciência e Tecnologia do Ceará \\ tiagoestevam@ifce.edu.br
}

José Borzacchiello da Silva Universidade Federal do Ceará borzajose@gmail.com

Resumo: Os estudos sobre a geografia do comércio e consumo iniciam-se aos poucos na ciência geográfica a partir das décadas de 1950 e 1960. Observa-se tal fato, principalmente, nas pesquisas realizadas pela geografia francófona a partir das investigações de Sporck, na Bélgica, Delobez e Beaujeau-Garnier, na França. Como também, pela geografia anglo-saxônica com estudos de Brian J. Berry. Nesse sentido, este artigo evidência a geografia do comércio e consumo sob uma perspectiva da geografia humana através dos diversos pressupostos, como por exemplo: a nova geografia, geografia crítica, virada cultural e o pós-estruturalismo geográfico. $\mathrm{O}$ artigo tem um caráter teórico englobando grandes baluartes da geografia do comércio e consumo. Em alguns momentos, mesmo com as divisões epistemológicas realizadas, parte-se da ideia que existe um emaranhando de concepções que, de certo modo, organizou-se na tentativa de ter uma visão mais abrangente e globalizante da geografia do comércio e consumo na sociedade da cultura do consumo. Não constitui-se necessariamente um estudo do percurso histórico, entretanto, observar-se alguns recortes temporais considerados relevantes para o entendimento dos pressupostos teórico-metodológicos que envolvem esse campo geográfico. Por fim, o artigo em seu bojo exprime o desencontro das temporalidades, os contrapontos entre o tradicional e o (pós) moderno, as continuidades e as descontinuidades do espaço urbano e metropolitano.

Palavras-chave: Comércio; Consumo; Cultura; Geografia.
Abstract: Studies on the geography of trade and consumption are slowly beginning in geographic science from the 1950s and 1960s. This fact is observed, especially in Fracophone geography surveys from the Sporck, Belgium, Delobez and Beaujeau-Garnier investigations in France.As well, by the Anglo-Saxon geography with studies of Brian J. Berry. In this sense, this article aims to understand the geography of commerce and consumption from a perspective of human geography through the geographic currents: new geography, critical, cultural and poststructuralist. The article has a theoreticalepistemological character. At times, even with the epistemological divisions, it starts from the idea that there is a "entanglement" of conceptions that, in a way, was organized in the attempt to have a more comprehensive and globalizing view of the geography of commerce and consumption in the society of consumer culture. It is not necessarily a study of the historical course, however, to observe some temporal cuts considered relevant for the understanding of the theoretical and methodological assumptions that involve this geographical field. Finally, the article in its bulge expresses the mismatch of temporalities, the counterpoints between the traditional and the (post) modern, the continuities and discontinuities of urban and metropolitan space.

Keywords: Commerce; Consumption; Culture; Geography. 


\section{INTRODUÇÃO}

A geografia do comércio e consumo é considerada por muitos autores uma temática irrelevante, sendo que, em determinados casos foi subestimada por muitos investigadores da geografia, visto que, alguns autores não observavam proveito no aprofundamento do tema. Entretanto, se nem todas as cidades são de acordo com a expressão de Henri Pirenne "filhas do comércio", nenhuma, porém, pode escapar da presença e influência do comércio. Como afirma Beaujeu-Garnier e Delobez (1977) nos primeiros estudos sobre a temática, o comércio aparece sem dúvida como o elemento que melhor traduz o tipo de sociedade que está em desenvolvimento. Há uma relação intrínseca entre comércio e cidade, isto é, o fato das atividades terciárias estarem relacionadas às mudanças urbanas, suas causas e necessidades.

Os estudos a respeito do comércio e consumo passam a ser incipiente na ciência geográfica a partir das décadas de 1950 e 1960. Com destaque, principalmente, aos estudos realizados pela geografia francófona com investigações de Sporck (1964), na Bélgica, Delobez e BeaujeauGarnier, na França e pela geografia anglo-saxônica com Brian J. Berry (1971). O nome de Berry está associado à Teoria dos Lugares Centrais, que permite entender a existência de um sistema urbano. Esta teoria é essencial para compreensão da organização das bases econômicas dos lugares sobre o comércio. A localização do comércio em meio urbano segue alguns procedimentos. Assim, os locais de conveniência dos meios de comunicação e os eixos de grande circulação são especialmente favorecidos, originado os chamados ribbon developments de Berry (1971). O varejo na cidade e as suas consequências econômicas, sociais e espaciais despertaram o interesse de pesquisadores em ciências sociais. A estrutura de cidades é enfatizada pelos geógrafos, Metton (1984) e Lemarchand (2011).

A respeito do papel desempenhado pelo consumo e seus desdobramentos articulado ao espaço e por sua vez a sociedade contemporânea, o pesquisador Carreras (1994) realizou e realiza importantes estudos sobre a geografia do comércio e consumo, sendo que, aborda novas perspectivas de análise ao conectar as atividades terciárias com a morfologia urbana e a dinâmica territorial no âmbito do consumo geral. Assim, busca uma nova geografia comercial urbana dando um papel de destaque ao consumo e seus significados na organização da sociedade. Seus estudos analisam o caso concreto da cidade de Barcelona e as mudanças ocorridas nela mediante o consumo.

Nos últimos anos, um diálogo produtivo se desenvolveu entre geógrafos do comércio e os geógrafos sociais preocupados com a espacialidade do consumo. Isso resultou em uma série de estudos que dão ênfase ao consumidor, as desigualdades socioespaciais evidentes no varejo. Nesta "nova geografia do comércio", determinadas preocupações tradicionais de teorização foram ampliadas para abranger considerações do consumo no mundo pós-moderno como uma atividade cultural e econômica (WILLIAMS, HUBBARD, BERKELEY, 2001).

No bojo desta discussão, Wrigley e Lowe (1996) consideram que a década de 1990 foi um período de crescente interesse entre os cientistas sociais, particularmente, aqueles da Europa que se debruçavam sobre a temática das culturas de consumo e os espaços de consumo do capital comercial. Simultaneamente, foi um período marcado na geografia humana pela emergência de um subcampo reconstruído da geografia do comércio, em que ocorreu o afastamento de seu foco tradicional em questões do comércio local e suaaproximação da geografia econômica e cultural. Esta "nova geografia do comércio", como assim a batizou Wrigley e Lowe (1996), foi caracterizada pelo envolvimento teórico e por uma apreciação do varejo e consumo, como uma das áreas mais fascinantes e desafiadoras de estudo em geografia humana contemporânea dada a importância do capital no comércio, consumo e espaço.

Neste contexto, a respeito da relação entre o capital financeiro e o capital comercial, Silveira (2009) adentra as relações do sistema financeiro e as novas formas organizacionais de empresas comerciais. Assim, na atualidade é necessáro o entendimento da oferta vultosa de crédito de instituições financeiras bancárias e não-bancárias (redes, franquias e outlet e dentre outras). Destaca-se, também, a abundância de formas de crédito pessoal que implica no aumento do consumo e, simultaneamente, o endividamento, a inadimplência e a insolvência. 
Goss (2006) destaca uma heterogeneidade de perspectivas teóricas e metodológicas da geografia do comércio e consumo, dentre as quais se destacam: Miller (1998), que desenvolve estudos sobre a cultura material; Klein (2000), com estudos sobre o efeito penetrante de marcas; Jackson (2000), sobre os aspectos inovadores do enraizamento cultural de práticas econômicas, por exemplo, na moda, varejo e publicação; Smith (2002), sobre a penetração da cultura de consumo em sociedades pós-socialista e em contextos pós-apartheid; Leslie (2002), que discute a importância de estudos sobre os funcionários no varejo de moda que também são consumidores dos modelos do produto que eles vendem; Clarke (2003), sobre as mudanças do tema do consumo e comércio; Ritzer (2004), que discute o "vazio" do consumidor na sociedade globalizada.

Nesse sentido, o artigo focará a geografia do comércio e consumo sob uma perspectiva da geografia humana através dos diversos pressupostos que vão desde a nova geografia até a chamada virada cultural ${ }^{1}$ e pós-estruturalismo geográfico.

Cachinho (2002) discute sobre a dificuldade do entendimento teórico-metodológico no campo da geografia do comércio e consumo, pois a pesquisa nesta área geográfica encontra-se em uma nova e diversificada perspectiva que vão desde estudos sobre antropologia e cultura passando por meio de estudos de negócios e marketing até a organização de questões sobre a economia e transnacionalizações industriais, assim torna-se bastante difícil definir o que seja precisamente geográfico, bem como do que seja o limite das outras áreas das ciências humanas, com isso observa-se que existe um desafio posto ao pesquisador de definir com clareza o que deseja apreender sobre tais questões, caso contário pode se perder diante de uma diversidade de estudo. O comércio pode ser entendido como um sistema aberto e dinâmico no contexto socioespacial que tem a atuação de diversos atores e agentes com papéis muito variados, sendo que, a sua reestruturação que ocorre no tempo e no espaço é compreendida através da ação dos vários agentes, ou seja, a teia de relações que estes estabelecem entre si e a mediação do contexto socioespacial onde ocorre as representações.

\section{PRESSUPOSTO TEÓRICO-METODOLÓGICO}

O conhecimento científico nasce possivelmente da busca do ser humano em ter um controle e poder de ação sobre os fenômenos e fatos, possuindo dessa maneira um papel ativo, e não uma posição de mero espectador daquilo que ocorre à sua volta. Nesse sentido, usando de suas potencialidades de racionalidade, cabe ao ser humano conceber de modo sistemático uma leitura, ou melhor, uma compreensão do mundo, utilizando os métodos de investigação e de aplicação.

Nesse contexto, é salutar destacar que um dos interesses do ser humano pela ciência se dá pela constante curiosidade de entender as relações que existem entre as ocorrências e o que existe por detrás das aparências. Nesse âmbito, podemos pensar na construção do espaço categoria de análise da pesquisa ora proposta - e na relação entre sociedade e natureza. É imprescindível que se busque não somente a aparência - o material -, mas também o imaterial, aquilo que somente a essência pode oferecer como perspectiva analítica do espaço.

O pressuposto teórico-metodológico desta pesquisa apoia-se em Henri Lefebvre, tendo como categoria analítica o espaço, mais especificamente a produção do espaço urbano-metropolitano. A investigação tem por base a leitura do trabalho de Henri Lefebvre (2002). O artigo foi desenvolvido observando o desencontro das temporalidades, os contrapontos entre o tradicional e o (pós) moderno, as continuidades e as descontinuidades do espaço urbano e metropolitano.

O espaço, ao se reproduzir, se materializa em vários momentos históricos ao mesmo tempo de maneira desigual, atribuindo diferenças entre esses espaços. Assim, compreender o espaço

\footnotetext{
${ }^{1}$ A virada cultural vai, entretanto, se desenhando, graças às orientações originalmente advindas da nova Geografia: debruça-se agora sobre a percepção do espaço e os vieses que ela introduz na disciplina, sobre os mapas mentais e as representações. (CLAVAL, 2012, p.14). [...] A virada cultural da geografia humana, que enfatiza o fato de que os processos sociais, econômicos ou políticos dependem das culturas onde eles atuam (CLAVAL, 2008, p. 28)
} 
implica raciocinar as diferenças espaciais e as características que se descobrem expostas ou implícitas no espaço.

\section{GEOGRAFIA HUMANA: ESTUDOS E CONTEXTOS DO COMÉRCIO E CONSUMO}

A questão teórico-metodológica da ciência geográfica é um ponto crucial de estudo e análise. Diante do avanço epistemológico da Geografia, observam-se diferentes concepções metodológicas de estudo. Desse modo, a geografia do comércio e consumo se apresenta com distintas concepções de acordo com o percurso geográfico vigente e/ou abordado pelo pesquisador. No âmbito da Geografia tradicional mais especificamente da geografia humana, o interesse pela abordagem do comércio e o consumo não era evidente.

Com o fim da segunda guerra mundial, com as transformações sociais, políticas e econômicas que influenciaram a sociedade, a organização territorial e o desenvolvimento científico e tecnológico, desse modo, o comércio teve uma evolução depois da segunda guerra mundial, destacando quatro significativos aspectos: especialização dos pontos de vendas, segmentação do mercado, desenvolvimento da localização e a emergência de novas polaridades (MÉRENNESCHOUMAKER, 2008).

Nas décadas de 1950 e 1960, os geógrafos neopositivistas procuraram colocar em prática os ditames do "método científico", ou seja, levantar e testar hipóteses, fazer a experimentações. A corrente do pensamento geográfico dessa base teórico-metodológica foi denominada de nova geografia, e vinculava-se aos interesses capitalistas expansionistas dentro da lógica bélica no conflito Estados Unidos (capitalista) e União Soviética (socialista).

Neste sentido, Delage e Fleury (2011) propõem o desenvolvimento metodológico para a análise das centralidades comerciais. A base de análise espacial é quantitativa, utiliza-se de algorítmos para escaneamento espacial. Os autores definiram como estudo de caso a cidade de Paris, os modelos são utilizados para classificação da geografia do comércio na trama comercial de Paris. Outro estudo que segue modelos matemáticos e estatísticos é de Baray (2011), este busca construir uma metodologia de aproximação espacial das atividades comerciais e dos serviços. Utiliza-se de cálculos de índice de complementaridade espacial das atividades, depois estrutura uma modelação de leis de dependência das atividades e suas formas, por fim tem como finalidade saber o comportamento e a hirerquização das atividades.

Silva (2014) realiza críticas às pesquisas com base matemática e estatística que se restringe aos estudos de localização, lógicas hierárquicas, classificações e modelações espaciais, pois, de acordo com o autor, tais estudos não conseguem resultar em respostas adequadas à reprodução do espaço de produção do espaço social. As análises dos geógrafos têm mostrado as transformações do dispositivo comercialestruturadas pelas seguintes dmensões: a primeira, a partir do papel inegável desempenhado pela organização dos territórios no desenvolvimento de estabelecimentos comerciais e a segunda através do impacto do varejo sobre a organização do território.

\section{PRODUÇÃO DO ESPAÇO SOCIAL: CIDADE E COMÉRCIO}

A partir da década de 1970 se desenvolveu a geografia crítica ou radical ligada às questões sociais e aos problemas político-ideológicos, fator de visibilidade para a geografia humana. A contradição e o conflito são características básicas do método dialético.

De acordo Overton (2010), os geógrafos têm se interessado quanto à produção social do espaço, buscando entender os significados e os valores que são atribuídos ao espaço e aos processos sociais, culturais e políticos. Nas últimas duas décadas, os geógrafos têm vindo a apreciar a maneira como o espaço é construído socialmente. A conceituação do espaço tem sido uma preocupação em termos de teoria geográfica, a exemplificar os trabalhos de: Soja (1993), Harvey (2005) e Massey (2012). Esses autores têm argumentado persuasivamente para o reconhecimento do espaço concebido de modo fluido, construído e reformado ao longo do tempo pelos aspectos sociais, culturais, econômicas e políticas. 
Hudson (2001, p. 282), tem estudado em particular o papel do capital em moldar espaço: "Dentro dos limites das relações sociais capitalistas, a produção do espaço está intimamente ligada aos imperativos da mercadoria produção. Capital procura moldar o espaço em seus interesses, informado pelas exigências da produção rentável".

$\mathrm{Na}$ análise Marxista, o espaço desenvolve-se devido à intensificação das contradições sociais e espaciais tanto nos países centrais como nos periféricos. Porém, é na obra de Lefebvre onde o espaço aparece efetivamente na análise marxista. Para o autor, o espaço é lócus da reprodução das relações sociais de produção e reprodução da sociedade. Lefebvre (2002) e Soja (1993) têm sugerido que o espaço é um artefato de imaginação e atividades humanas. As noções de espaço estão ligadas ao que experimentamos, valorizamos e desenvolvemos nos lugares em que vivemos.

Henri Lefebvre (2002) afirma que o espaço é, ao mesmo tempo, um produto e condicionador das relações sociais. A prática socioespacial é bastante pertinente para os estudos do campo da geografia do comércio e consumo imbricados na geografia urbana. Sobre o consumo, com forte base crítico- marxista, o autor Milton Santos (2009) discute sobre a possibilidade de "defender o consumismo", em que considera que pode ser uma hábil manobra política ou uma forma de oportunismo sofisticado, com o qual provavelmente se pode conquistar o povo e ganhar o poder.

As formas do comércio se mostram como complexidades socioespaciais, pois são produtos históricos, desenvolvidos ao longo da existência humana em sua busca constante de meios e respostas rápidas e eficazes para satisfação de suas necessidades de vida, que no caso da sociedade capitalista são, antes de qualquer coisa, as necessidades do capital e é evidente que isso não se faz sem as pessoas, mas o objetivo final é a reprodução ampliada do capital (CLEPS, 2000; PACHECO, 2012).

O comércio tem sido cada vez mais encarado como uma verdadeira função urbana estreitamente relacionada com processos de urbanização ficando evidenciado valências e dinâmicas que extravasam a mera função econômica. As atividades comerciais sempre estiveram associadas ao desenvolvimento urbano. Assim, o estudo sobre o setor terciário possibilita compreender as transformações ocorridas no espaço urbano.

Vivencia-se uma cultura da apreciação pelo materialismo, nesse contexto, há uma inserção das mercadorias no dia-a-dia das pessoas, ultrapassando as barreiras da relação compra/consumo, estendendo a vida e a durabilidade de seus pertences a uma rotina diária criando um verdadeiro elo entre consumidor e produto. Nesse contexto, as atividades comerciais podem, assim, ser associadas a um renascimento da vida pública, bem como com as tendências para a privatização e homogeneização social e fragmentação espacial (KÄRRHOLM, 2009).

Ao considerar as mudanças da economia urbana, nota-se um novo quadro com o processo de globalização e de reestruturação produtiva do capital. Neste quadro as atividades terciárias passam a ter um papel relevante, com isso, torna-se pertinente o estudo sobre a temática do comércio e do consumo no âmbito geográfico.

Neste contexto, o urbano ganha notoriedade nas novas leituras da geografia do consumo e comércio. Assim, observam-se as relações entre comércio e o urbano na tentativa de serem vistos através da nova geografia econômica e cultural e de comércio. (WRIGLEY; LOWE; CURRAH, 2002).

A estrutura do comércio tem acompanhando ou antecipado alterações espaciais e sociais das cidades, por exemplo: a mudança nas perifeiras urbanas incentivada pelo comércio é um movimento que não pode ser separado da população e do surgimento de novos dispositivos de polaridades econômicos e culturais. Este movimento espacial não pode ser considerado em si, mas é também um aspecto de transformação social de grandes empresas mundiais que moveram a formação da sociedade de consumo (GONÇALVES; SILVA, 2017).

O comércio, em sua longa história, sempre fez parte da própria razão de ser das cidades, ou seja, o comércio promove também a existência da cidade, justifica em boa parte da sua organização interna, contribui para explicar os inúmeros movimentos que se desenvolvem no seu interior. Essas relações entre a cidade e o comércio são dinâmicas e a cidade vai se tornando 
produto das decisões e das práticas de diversos atores, entre eles os comerciantes, os consumidores, os promotores imobiliários e os produtores/fabricantes. Além de ser produto de toda esta dinâmica comercial a cidade, pode ser considerada condição e meio para que essas práticas continuem a se realizar. Daí a importância da dimensão espacial na análise do comércio e do consumo (ORTIGOZA, 2008; PINTAUDI, 2004, VARGAS 2001). O comércio surge como parte integrante das condições de vida da sociedade e também como produto desta, numa relação dialética e histórica.

\section{PERSPECTIVAS DA DIMENSÃo CULTURAL E RELACIONAL NA GEOGRAFIA dO COMÉRCIO E CONSUMO}

A abordagem cultural na geografia tem denotado um nítido interesse pelo pensamento pósmoderno, passou a dar mais atenção às questões anímicas e ontológicas dos seres humanos. Não se tratava mais de estudar a diversidade cultural com base nos seus conteúdos materiais, mas de admitir que a cultura esteja intimamente ligada ao sistema de representações, de significados, de valores que criam uma identidade que se manifesta mediante construções compartilhadas socialmente e expressas espacialmente, o espaço passa a ser concebido como espaço vivido, ou seja, como um espaço de vida, construído e representado por seus atores sociais, um território com suas dimensões sociopolíticas (controle, apropriação) e culturais (significado, identidade). Além disso, as temáticas relacionadas à religião, à percepção ambiental, às representações sociais, à identidade espacial e à interpretação de texto (literatura, música, pintura, cinema) destacam-se entre outras que foram retomadas ou emergiram diante das transformações sociais, políticas, econômicas e culturais vivenciadas pela sociedade contemporânea (ZANNATA, 2008).

Neste sentido, o consumo na dimensão cultural pode ser entendido como um processo global intersubjetivo, conjugando emoções, sentimentos, manifestações à existência humana. $O$ consumo é considerado um processo psicossocial e simbólico. Assim, o destaque maior é dado ao consumidor em detrimento do comerciante e distribuição dos objetos. A ideia de espacialidade adquire dimensões significativas e a distância define relações de proximidade ou desumanização. Os lugares do comércio se dão a partir da percepção e vivência da cidade através dos sentimentos e emoções das pessoas associados aos lugares de varejo (CACHINHO, 1999, 2002). Parafraseando Canclini (1999, p.39): "As lutas de gerações a respeito do necessário e do desejável mostram outro modo de estabelecer as identidades e construir a nossa diferença. Vamos nos afastando da época em que as identidades se definiam por essências a-históricas". Alguns estudiosos têm atuado no sentido de transcender dualismos, muitas vezes, erguidos entre comércio (econômico) e cultura que se tornou uma espécie de mantra para os novos geógrafos de consumo (LOWE, WRIGLEY, 2000). Seguindo essas ideias Jackson (2002) afirma que ocorre um esforço para estabelecer uma "economia cultural“. Sobre a "economia cultural", em se tratando dos novos estudos da geografia do consumo, é a chamada emergente "cultura comercial", uma forma híbrida que transcende dualismos convencionais (JACKSON, 2000). Para uma nova geografia do consumo, Jackson ataca explicitamente o 'vazio' de tentativas marxistas para "desvendar" o fetichismo de mercadorias.

O antropólogo Daniel Miller (2007) parece reconhecer uma falta de vontade da nova literatura sobre o consumo de se envolver com a "complexidade" do conceito de fetichismo de Marx como uma relação reificada com as coisas. Miller (2001, p. 227) defende "um encontro adequado com estudos atuais de consumo e consumidores suficientemente diferenciados para ser devidamente orientados para o complexo processo contraditório do consumo". Considera viável estudar o real desejo do consumo e do consumidor por meio das lentes etnográficas (MILLER et al. 1998).

A geografia do consumo deve muito à sociologia do consumo. Neste campo científico aparecem cientistas sociais que questionam a finalidade social e política do consumo. Entram neste bojo os chamados pós-modernos Baudrillard (1995), Lyotard (2011) Maffesoli (1988) ou hiper-modernos Lipovestky (2004). O pensamento pós-moderno colabora para compreender o consumo além do convencional ou distintivo, contudo, a partir de uma afirmação de consumo através da identidade, sem excluir a dimensão social relacionada à variável econômica. Para a 
geografia, não surpreendentemente, o entedimento desses autores conduz a considerar o lugar do comércio como lugares de expressão das novas formas sociais das sociedades pós-modernas.

Neste arcabouço o pesquisador norte-americano Miller (2013) realiza um estudo desafiador sobre o Abasto Shopping, em Buenos Aires, Argentina, em uma perspectiva etnográfica, contudo com uma base dialética. O autor considera que, o estudo dos shoppings na dinâmica urbana, através da dialética socioespacial é um guia teórico útil. A concepção dialética do espaço, identidade e política possibilita a exploração do papel da afetividade na produção do espaço do consumidor, bem como do mundo da representação, incluindo, ideologia, discurso, significação e significado é obviamente importante na geração de assuntos do comércio e consumo, todavia não é o único registro da experiência pela qual o poder pode operar. De acordo Lemarchand (2011) tem sido pouco estudado o consumo nesta perspectiva pelos geógrafos franceses. No entanto, o estudo dos lugares emblemáticos de consumo, tais como armazéns ou centros comerciais, têm tido especial atenção por parte dos anglo-saxônicos como os estudos de Jackson e Thrift (1995).

No contexto da virada cultural as fronteiras entre as ciências humanas tornaram-se mais permeáveis, de modo que os temas fossem renovados, apresentando um novo vocabulário e aparato conceitual, desenvolvendo novas metodologias, portanto, as fronteiras epistemológicas entre as ciências humanas aparecem pela primeira vez como discutíveis ao apresentar três tipos de viradas que possuem relação intrínseca, a destacar, virada linguística, virada espacial e virada cultural da geografia, ocorrida nos anos 1980. Sobre a virada cultural da geografia tem um sentido bem expressivo, pois as áreas e subáreas da geografia apresentavam-se suas fronteiras com bastante rígidez. Assim, a virada cultural sginifica de acordo com Claval (2011) uma totalidade dos saber geográfico tendo uma dimensão cultural: eles são relativos a uma época, a um lugar ou a uma área.

Na Geografia anglo-saxônica, as tendências contemporâneas mostram que a virada cultural sem a pretensão de um método ou uma verdade científica representa um novo conselho com base em estabelecer argumentos e verdades questionáveis. Assim, apesar de suas limitações, de acordo com Lemarchand (2011) a virada cultural, inegavelmente, questionou a prática científica e a forma de fazer geografia e, portanto, assim, a geografia do comércio passa, por sua vez, a ter um novo olhar sobre o ato de consumir.

Cachinho (2002) apresenta três dimensões sobre o caminho etnogeográfico em estudos do consumo e comércio, de forma sucinta, a primeira dimensão analisa as formas e funções com destaque aos shopping centers, aos espaços de consumo na pós-modernidade; a segunda dimensão diz respeito a subjetividade, a relação entre os objetos e lugares de consumo, em que o consumo atua como uma ponte entre o indivíduo e sua experiência no espaço. $E$ a terceira vertente dialoga sobre o espaço e os lugares que definem o mundo dos consumidores, a publicidade que atua na alteração dos aspectos da realidade e o consumo na defnição de identidades.

Com o estudo do consumo, os geógrafos têm procurado explicar como e o porquê da interação da economia e cultura, do simbólico e do material, demonstrando como os significados e expressões de consumo nos lugares estão por sua vez ligados a outras áreas geográficas e escalas; isso permite contribuir para a compreensão crítica da relação entre espaço e sociedade (MANSVELT, 2005).

Nos estudos de Mansvelt (2010) destaca-se uma dimensão de pesquisas em geografias relacionais que enfatiza como os indivíduos se tornam consumidores e analisa o consumo em múltiplos contextos sociais e espaciais. O consumo é realizado e expresso através de interações complexas entre coisas materiais e imateriais, organizações e estados com diversas formas práticas. A cultura do consumo não pode ser reduzida apenas a mercadorias, a lojas ou aos consumidores, mas deve ser entendida em termos de relacionalidade; como um circuito recursivo (CREWE, 2003).

Em uma perspectiva pós-estruturalista, o espaço é relacional e culturalmente produzido, vivido fisicamente, concebido socialmente e percebido individualmente. Cachinho (2002) afirma que os pós-estruturalistas, estruturacionistas e pós-modernos tem em comum a crítica em relação ao projeto da modernidade. Em uma base da relatividade o espaço encontra-se em constante 
transformação e reconfiguração correspondendo a um elemento essencial de manifestação de identidade, sendo, também objeto de diversas interpretações e discussão de ideias. Massey (2012) procura articular a imaginação sobre espaço no quadro de referências mais amplas do pensamento científico e suas transformações. É preciso refletir sobre o espaço e repensar a globalização, lugar, cidade, nesse bojo repensar o papel do comércio e consumo nas metrópoles contemporâneas.

O espaço, na atualidade, é visto de modo interligado com o tempo - não é estático, neutro, imóvel e gélido. Massey (2012) propõe uma nova conceituação sobre espacialidade, afirma que, nossos sentidos não conseguem perceber a espacialidade em potencial. A base para tal conceituação está na filosofia geográfica e como suas hipóteses podem ter limitado nossa imaginação do espaço.

Assim, o espaço deverá ser compreendido na multiplicidade em que vivemos, pode ser visto como um produto de inter-relações, como uma esfera de multiplicidade, espaço como algo sempre em construção. Na modernidade, os espaços eram separados e colocados em uma mesma linha histórica, a preocupação era com o tempo, com o progresso dos "atrasados". Na pósmodernidade, a globalização, o espaço de fluxos, a ampliação da conexão entre os lugares apagou as diferenças históricas, colocando todos no mesmo presente.

\section{CONSIDERAÇÕES FINAIS}

O comércio na cidade, as suas consequências econômicas, sociais e espaciais como uma linha de interesse por parte de muitos pesquisadores em ciências sociais e em especial em geografia foi abordada neste artigo. Verificou-se que as atividades terciárias são na atualidade o principal motor econômico em diversas regiões do planeta tanto no mundo desenvolvido quanto no mundo em via de desenvolvimento. Deste modo, essas atividades agem muitas vezes como criadora e força motora do crescimento urbano. Assim, na contemporaneidade, os estudos do comércio e do consumo estão se tornando cada vez mais importante.

No âmbito atual da geografia do comércio e consumo houve um avanço em diferentes rumos quando comparado com os primeiros estudos realizados pela nova geografia, sendo que, na perpectiva contemporânea se volta às transformações do mundo flexível e à sociedade da cultura do consumo.

Recentemente, o tema do consumo e comércio adquire especial reconhecimento, além do mais, os geógrafos urbanos e econômicos acreditam que mais estudos precisam ser feitos para entender o papel do consumo e comércio na formação da economia urbana contemporânea.

Considerou-se, assim, o consumo como sendo um ato econômico, entretanto com implicações socioculturais de grau variado no qual se buscou abordagens urbanas e econômicas amplas e diversas. O entendimento do comércio perpassa o consumo e produção, a intersecção entre consumo e a economia urbana através da ótica cultural-simbólica se traduz em paisagens econômicas da vida urbana. Assim, o consumo é o presente e o futuro das economias urbanas, e é a força motriz por trás da forma urbana.

É relevante apontar como os estudos a partir da virada cultural propiciaram e validaram a análise cultural para compreender melhor o papel atual desempenhado pelos lugares e áreas comerciais em uma sociedade onde a prática consumista tornou-se uma prática de identificação.

Nesse sentido, o espaço pode ser pensado também como produto de inter-relações, de forma que não há um ponto de partida original a ser recuperado ou uma posição que seja anterior à relação. Em sua conceituação Soja (1993) destaca que o espaço e a espacialidade são fundamentais para a constituição e devir da sociedade. Destarte, o espaço que incluía as noções de práticas espaciais e representações de espaço, acresce seu "terceiro espaço", o de "espaços de representação".

Assim, o espaço do consumo e o consumo do espaço são percebidos não apenas como socialmente construído, mas também como um ato cultural e econômico a ser consumido. Nesta vertente, presenciam-se estudos contemporâneos sobre o comércio e consumo em diversos 
países, como por exemplo: Portugal, Espanha, Estados Unidos, Canadá, Nova Zelândia, Austrália, Suécia e Reino Unido. Imbuídos destas reflexões buscou-se relacionar adequadamente as novas perspectivas sociais e culturais da geografia do comércio e consumo através de uma apreciação da situação das forças econômicas e suas repercussões nas relações culturais no mundo globalizado.

Por fim, existe um desafio permanente que se dá no sentido do pesquisador romper com os paradigmas positivistas da ciência moderna, em que cada ciência deve ficar fechada em sua caixa de pandora, ou seja, o investigador deve buscar ao máximo a interdisciplinaridade, ou quem sabe, a transdiciplinaridade, sendo que estes aspectos de construção do pensar sobre o comércio e consumo são bastante complexos, contudo poderá conduzir a respostas mais profícuas sobre a realidade contemporânea.

\section{REFERÊNCIAS}

BARAY, J. Complémentarité spatiale: Modélisation d'um réseaus géographique de commerces et de services de proximité. Bulletin de la Societé géographique de Liège,56, p. 23-34, 2011.

BAUDRILLARD, J.A sociedade de Consumo. Lisboa: Edições 70, 1995.

BEAUJEU-GARNIER, J.;DELOBEZ, A. Géographie du commerce. Paris: Masson, 1977.

BERRY, B. J. L. Géographie des marchés et du commerce de détail.Paris: A. Colin, 1971.

$\mathrm{CACHINHO}, \mathrm{H}$. O comércio retalhista português: pós-modernidade, consumidores e espaço. Lisboa: Gabinete de Estudos e Prospectiva Econômica do Ministério da Economia, 2002.

CANCLINI, N. G.. Consumidores e cidadãos: conflitos multiculturais da globalização. Rio de Janeiro: Editora UFRJ, 1999.

CARRERAS, C. Os novos espaços de consumo em Barcelona. Finisterra, XXIX, 57, p.103-117, 1994.

CLARKE, D.B. The consumer society and the postmodern city. London and New York: Routledge, 2003.

CLAVAL, P. Geografia Cultural: Um balanço. Revista Geografia Londrina, v.20, n.03, p.05-24, set/dez, 2011.

CLAVAL, P. A geografia cultural no Brasil. In: BARTHE-DELOIZY, F.; SERPA, A., (Org). Visões do Brasil: estudos culturais em Geografia [online]. Salvador: EDUFBA; Edições L'Harmattan, 2012.

CLAVAL, P. Uma, ou algumas, abordagem (ns) cultural (is) na geografia humana? In: SERPA, Ângelo (Org.). Espaços Culturais: vivências, imaginações e representações. Salvador: EDUFBA, 2008.

CLEPS, G.D.G. A origem e o desenvolvimento do comércio atacadista de Uberlândia (MG). Revista Sociedade \& Natureza, Uberlândia, v.12, n.23, p.5-45, 2000.

CREWE, L. Geographies of retailing and consumption: markets in motion. Progress in Human Geography, 27 (3), p. 352-62, 2003.

DELAGE, M.; FLEURY, A. Centralités commerciales et logique hiérarchique en milieu urbain dense. Une démarche renouvelée d'analyse spatiale appliquée au cas de Paris. Bulletin de la Societé géographique de Liège, 56, p.7-21, 2011.

GONÇALVES, T.; SILVA. J.B. da. O papel dos shopping centers da Região Metropolitana de Fortaleza: centralidades, comércio e consumo. III Seminário regional comércio, consume e cultura nas cidades. Anais... Sobral-CE, 2017.

GOSS, J. Geographies of comsumption: the work of consumption. Progress in Human Geography,30, 2, p. 237-249, 2006.

HUDSON, R. Producing Places.: New York: Guilford Press, 2001. 
JACKSON, P.; THRIFT, N.J. Geographies of consumption. In: MILLER, D. (org.) Acknowledging consumption: a review of new studies. London: Routledge,1995.

JACKSON, P. Introduction: consumption, audiences and commercial culture. In: JACKSON, P.; LOWE, M.; MILLER, D.; MORT, F. (editors), Commercial cultures: economies, practices, spaces, Oxford and New York: Berg, 2000.

JACKSON, P.Commercial cultures: transcending the cultural and the economic. Progress in Human Geography,26(1), p. 3-18, 2002.

HARVEY, D. A produção capitalista do espaço. São Paulo: Annablume, 2005.

KÄRRHOLM, M. To the rhythm of shopping-on synchronisation in urban landscapes of consumption. Social \& Cultural Geography, v. 10, n. 4, Junho, 2009.

KLEIN, N. No logo: taking aim at the brand bullies. New York: Picador, 2000.

LEFEBVRE, H. A revolução Urbana. Belo Horizonte: Editora UFMG, 2002.

LEMARCHAND, N. Nouvelles approches, nouveaux sujets en géographie du commerce. Une géographie renouvelée par l'analyse culturelle. Géographie et cultures, 77, 2011.

LESLIE, D. Gender, retail employment and the clothing commodity chain. Gender, Place and Culture, 9(1), 61-76, 2002.

LIPOVETSKY, G. Os Tempos Hipermodernos. Mário Vilela (trad.), São Paulo: Barcarolla, 2004.

LOWE, M.; WRIGLEY, N. Retail and the urban. Urban Geography, 21, 7, p. 640-653, 2000.

LYOTARD, J.-F. A condição pós-Moderna. Trad. Ricardo Corrêa Barbosa. Rio de Janeiro: José Olympio, 2011.

MAFESSOLI, M. Les temps de Tribus. Le déclin de l'individualisme dans les sociétés de masse, Paris: Méridiens Klincksieck, 1988.

MANSVELT, J. Geographies of consumption, Londres: Sage Publications, 2005.

MANSVELT, J. Geographies of consumption: engaging with absent presences. Progress in Human Geography,34(2), p.224-233, 2010.

MASSEY, Doreen. Pelo espaço: uma nova política da espacialidade. Tradução de Hilda Pareto Maciel e Rogério Haesbaert. Rio de Janeiro: Bertrand Brasil, 2012.

MÉRRENE-SCHOUMAKER, B. Géografies des services et des commerces. Rennes: Presses universitaires de Rennes, 2008.

METTON, A. (dir.).Le commerce urbain français. Paris, PUF, collection UNIVERSITÉ D'ORLÉANS, 1984.

MILLER, D.; JACKSON, P.; THRIFT, N.; HOLBROOK, B. and ROWLANDS, M. Shopping, place, and identity. London and New york: Routledge, 1998.

MILLER, D.The poverty of morality. Journal of Consumer Culture,1(2), 225-43, 2001.

MILLER, D. Consumo como cultura material. Horizontes Antropológicos, Porto Alegre, ano 13, n. 28, p. 33-63, jul./dez. 2007.

MILLER, J. C. The Spatial Dialectics of Modernity and Retail Affect at Abasto Shopping, Buenos Aires, Argentina. Urban Geography .v. 34, n. 6, 843-863, 2013.

ORTIGOZA, S. A. G. A metrópole pós-moderna como centro do consumo: notas sobre São Paulo (Brasil) e Lisboa (Portugal). Revista Estudos Geográficos. Rio Claro, v. 6, n. 1, 2008.

OVERTON, J. The consumption of space: Land, capital and place in the New Zealand wine industry. Geoforum, 41, p. 752-762, 2010.

PACHECO, S.M.M. Reflexões sobre a atualidade do conceito de subcentro em áreas consolidadas na metrópole carioca. In: PACHECO, S. M. M; MACHADO, M. S. (Org.) Globalização, políticas públicas e reestruturação territorial. Rio de Janeiro: 7 Letras, 2012. 
PINTAUDI, S. M. Para uma leitura das formas de comércio varejista na cidade. Revista Cidades. Presidente Prudente: Grupo de Estudos Urbanos, v. 7, n. 11, 2004.

RITZER, G. The globalization of nothing. Thousand Oaks, CA, and London: Pine Forge Press, 2004.

SANTOS, M. Pobreza Urbana. São Paulo: Editora da Universidade de São Paulo, 2009.

SILVA, C. H. C. da. Estudos sobre o comércio e o consumo na perspectiva da geografia urbana. Geosul, Florianópolis, v. 29, n. 58, p 149-178, jul./dez. 2014.

SILVEIRA, M.L. Finanças, consumo e circuitos da economia urbana na cidade de São Paulo. Caderno CRH, v.22, n.55, p.65-76, 2009.

SMITH, A. Culture/economy and spaces of economic practice: positioning households in postcommunism. Transactions of the Institute of British Geographers, NS 27, 232-50, 2002.

SOJA, E.W. Geografias pós-modernas: a reafirmação do espaço na teoria social crítica. Rio de Janeiro: Jorge Zahar, 1993.

SPORCK, J.-A., 1964, Étude de la localisation du commerce de détail. Aspects méthodologiques, Bulletin de la Société Belge d'Études géographiques, $X X X I I I, n^{\circ} 1$, p. 63-70.

VARGAS,H. C. Espaço terciário: o lugar, a arquitetura e a imagem do comércio. São Paulo: SENAC, 2001.

WILLIAMS, P.; HUBBARD, P.; CLARK, D.; BERKELEY, N. Consumption, exclusion and emotion: the social geographies of shopping. Social \& Cultural Geography ,v.2, n.. 2, 2001.

WRIGLEY, N.; LOWE, M. Retailing, Consumption and Capital: Towards the New Retail Geography. Harlow, UK: Longman, 1996.

WRIGLEY, N.; LOWE, M.; CURRAH, A. Retailing and E-Tailing. Urban Geography,2002, 23, 2, p. 180-197, 2002.

ZANATTA, B. A. A Abordagem Cultural na Geografia. Temporis(ação) (UEG), v. 1, p. 249-262, 2008. 